\title{
Unsteady aerodynamic forces on small-scale wings: experiments, simulations and models
}

\author{
Steven L. Brunton*, Clarence W. Rowley ${ }^{\dagger}$ \\ Princeton University, Princeton, NJ 08544 \\ Kunihiko Taira ${ }^{\ddagger}$, Tim Colonius ${ }^{\S}$ \\ California Institute of Technology, Pasadena, CA, 91125 \\ Jesse Collins ${ }^{\top}$, David R. Williams" \\ Illinois Institute of Technology, Chicago, IL, 60616
}

\begin{abstract}
The goal of this work is to develop low order dynamical systems models for the unsteady lift and drag forces on small wings in various modes of flight, and to better understand the physical characteristics of unsteady laminar separation. Velocity field and body force data for a flat plate at static angle of attack and in sinusoidal pitch and plunge maneuvers are generated by 2D direct numerical simulations using an immersed boundary method at $R e=100$. The lift of a sinusoidally plunging plate is found to deviate from the quasi-steady approximation at a reduced frequency of $k=0.5$ over a range of Strouhal numbers. Lagrangian coherent structures illustrate formation and convection of a leading-edge vortex in sinusoidal pitch and plunge. A phenomenological ODE model with three states is shown to reproduce the lift on a flat plate at a static angle of attack above the stall angle. DNS for a 3D pitch-up maneuver of a rectangular plate at $R e=300$ shows the effect of aspect ratio on vortical wake structure and lift. Wind tunnel experiments of a wing in single pitch-up and sinusoidal pitch maneuvers are compared with a dynamic model incorporating time delays and relaxation times to produce hysteresis.
\end{abstract}

\section{Introduction}

Most models used for flight control rely on the quasi-steady assumption that lift and drag forces depend on parameters such as relative velocity and angle of attack in a static manner. While these models work well for conventional aircraft, they do not describe the unsteady laminar separation characteristics of flows over small, agile wings, for which the dynamics, or time history, of these parameters become important. Small wings flying at low Reynolds numbers (say, $\operatorname{Re}<20,000$ ) are susceptible to dynamic stall in the presence of large gusts and rapid maneuvers. Dynamic stall occurs when the effective angle of attack changes rapidly so that a leading-edge vortex forms, provides temporarily enhanced lift and decreased pitching moment, and then sheds downstream, resulting in stall ${ }^{1}$. This phenomenon is well known in the rotorcraft community ${ }^{2}$ since it is necessary to pitch the blade down as it advances and pitch up as it retreats to balance lift in forward flight. It has been shown that certain insects ${ }^{3,4}$ and birds ${ }^{5}$, flying at comparable Reynolds numbers, stabilize the leading-edge vortex to provide greater than expected lift, making this an interesting area for model-based control in the arena of micro-air vehicles ${ }^{6}$.

To better study the mechanism underlying transient lift and drag forces due to a disturbance, it is vital to know on what time scales the disturbance excites unsteady effects, rendering the quasi-steady approximation invalid. Knowledge of relevant time scales and the underlying flow physics is essential for constructing a hierarchy of models for the unsteady aerodynamics. It is possible to obtain reduced order models by projective methods such as balanced truncation or proper orthogonal decomposition. ${ }^{7} \mathrm{~A}$ bottom-up approach involving simple nonlinear models which are phenomenologically similar to observed flows may lead to more natural control design, and this is the approach we pursue here. By analyzing canonical maneuvers such as a plate in pure pitch or pure plunge, we hope to discover physically relevant state variables and how they relate to the generation of lift, drag and moment.

The organization of the paper is as follows: in Section II., we describe the results of 2D simulations of a lowReynolds number plunging plate; in Section III., we use Lagrangian Coherent Structures to visualize the unsteady

\footnotetext{
* Graduate Student, Mechanical and Aerospace Engineering, Student Member.

$\dagger$ Associate Professor, Mechanical and Aerospace Engineering, Member.

$\ddagger$ Graduate Student, Mechanical Engineering, Student Member.

$\S$ Professor, Mechanical Engineering, Member.

๑ Graduate Student, Mechanical, Materials, \& Aerospace Engineering, Student Member.

" Professor, Mechanical, Materials, \& Aerospace Engineering, Associate Fellow.
} 
flow in these simulations; and in Section IV. we present a simple phenomenological model for the dynamics of the flow, valid near the point where unsteadiness first appears. Next, we present some preliminary results of 3D simulations in section V., and finally we present some wind tunnel experiments on a pitching wing with semi-circular planform, and comparison with an empirical model previously proposed by Goman and Khrabrov ${ }^{8}$.

\section{Unsteadiness in 2D pure plunge maneuvers}

To understand the onset of unsteady effects in a moving airfoil, we initially investigate the relationship between reduced frequency and the lift of a flat plate sinusoidally plunging over a range of Strouhal numbers.

Using a fully resolved direct numerical simulation based on an immersed boundary method developed at Caltech $^{9,10}$, it is possible to construct incompressible flow experiments at low Reynolds numbers $(<500)$ and obtain velocity snapshots of the flow field. Here, we describe the results of 2D simulations performed at Reynolds number 100 on a spatial domain of $4 \times 4$ (nondimensionalized by the chord length), with grid resolution $200 \times 200$. We computed the steady-state lift of a flat plate for angle of attack $\alpha \in[0,90]$, discovering a Hopf bifurcation in lift at $\alpha_{c} \approx 28^{\circ}$. For $\alpha>\alpha_{c}$, a stable limit cycle develops, corresponding to periodic vortex shedding from the leading and trailing edge.

It is well known ${ }^{11,12}$ that under the quasi-steady approximation, a slowly plunging airfoil with center of mass motion $h(t)=(A / 2) \sin (2 \pi f t)$ may be related by a geometric transformation (at each instant) to a static airfoil at an effective angle of attack:

$$
\alpha_{\text {eff }}=\tan ^{-1}\left(\frac{\dot{h}}{U}\right) \quad U \equiv \text { free stream velocity }
$$

The maximum magnitude of $\alpha_{\text {eff }}$ for a plunging flat plate at $0^{\circ}$ angle of attack bias is determined by the Strouhal number, $\mathrm{St}=f A / U$, by $\alpha_{\mathrm{eff}}^{\max }=\tan ^{-1}(\pi \mathrm{St})$. To determine when the quasi-steady approximation breaks down, we ran a number of simulated plunging maneuvers at fixed Strouhal number, but with different reduced frequency $k=2 \pi f c /(2 U)$. Figures 1 and 2 show the observed lift curve against the quasi-steady approximation. Over a moderate range of Strouhal numbers, St $\in\{.032, .064, .127\}$, the observed lift curve begins to deviate from the $C_{L}\left(\alpha_{\text {eff }}\right)$ curve at a reduced frequency of $k \approx 0.5$, at which point the phase of the effective lift curve begins to lag the actual lift curve. Also, for each Strouhal number, at $k \approx 1$, the phase difference reaches a maximum of $-\pi / 2$ at which point doubling the frequency results in doubling the lift. This suggests that there are two time-scales on which the quasi-steady model breaks down in the case of pure plunge. For larger Strouhal numbers, $S t \geq .255$, even low reduced frequency maneuvers deviate from the quasi-steady model since the range of $\alpha_{\text {eff }}$ includes the Hopf bifurcation at $\alpha \approx 28^{\circ}$ seen in Figure 6 . Similar experiments can be done with pure pitching maneuvers by relating the pitching motion to an effective camber.
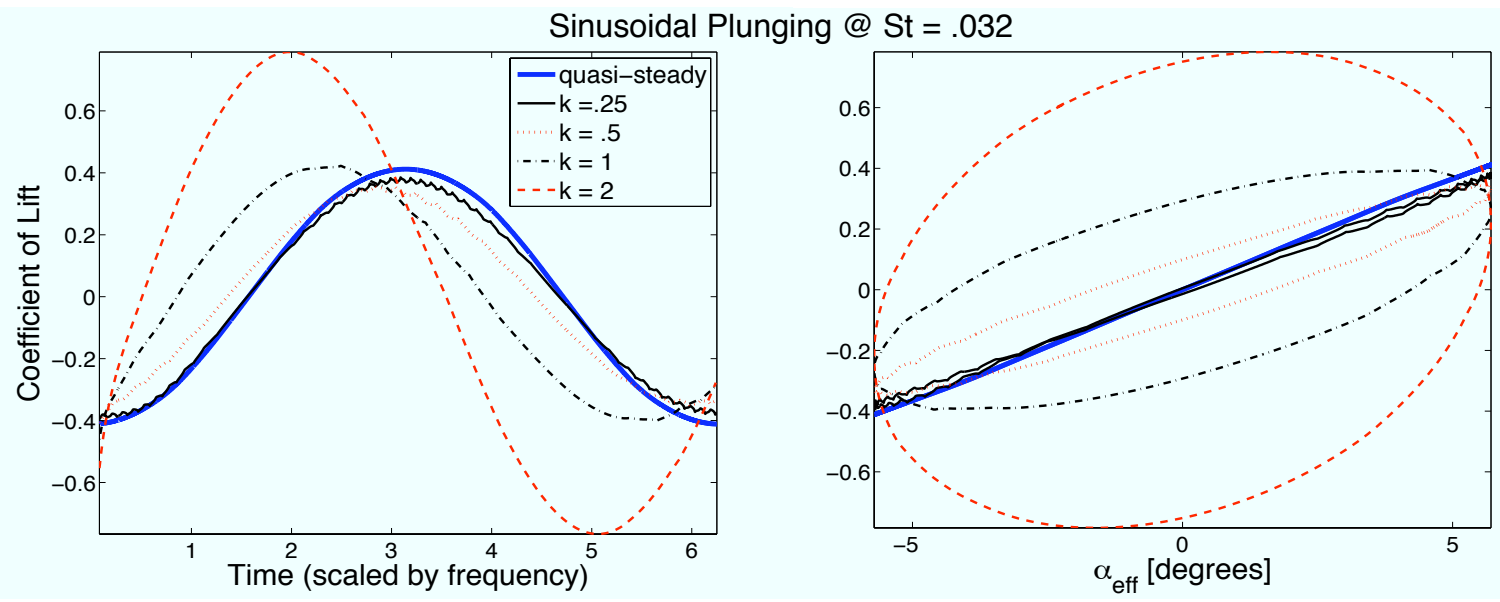

Figure 1. Comparison of actual $C_{L}$ vs. $\alpha_{\text {eff }}$ approximation for plate in sinusoidal plunge maneuver at $S t=.032$. Center of mass of plate is $h(t)=(A / 2) \sin (2 \pi f t)$; Strouhal number is $\mathbf{S t}=f A / U$; reduced frequency is $k=2 \pi f c /(2 U)$. Deviation from quasi-steady occurs at $k \approx 0.5$. (Left) $C_{L}$ vs. time (Right) $C_{L}$ vs. $\alpha_{\text {eff }}$. 

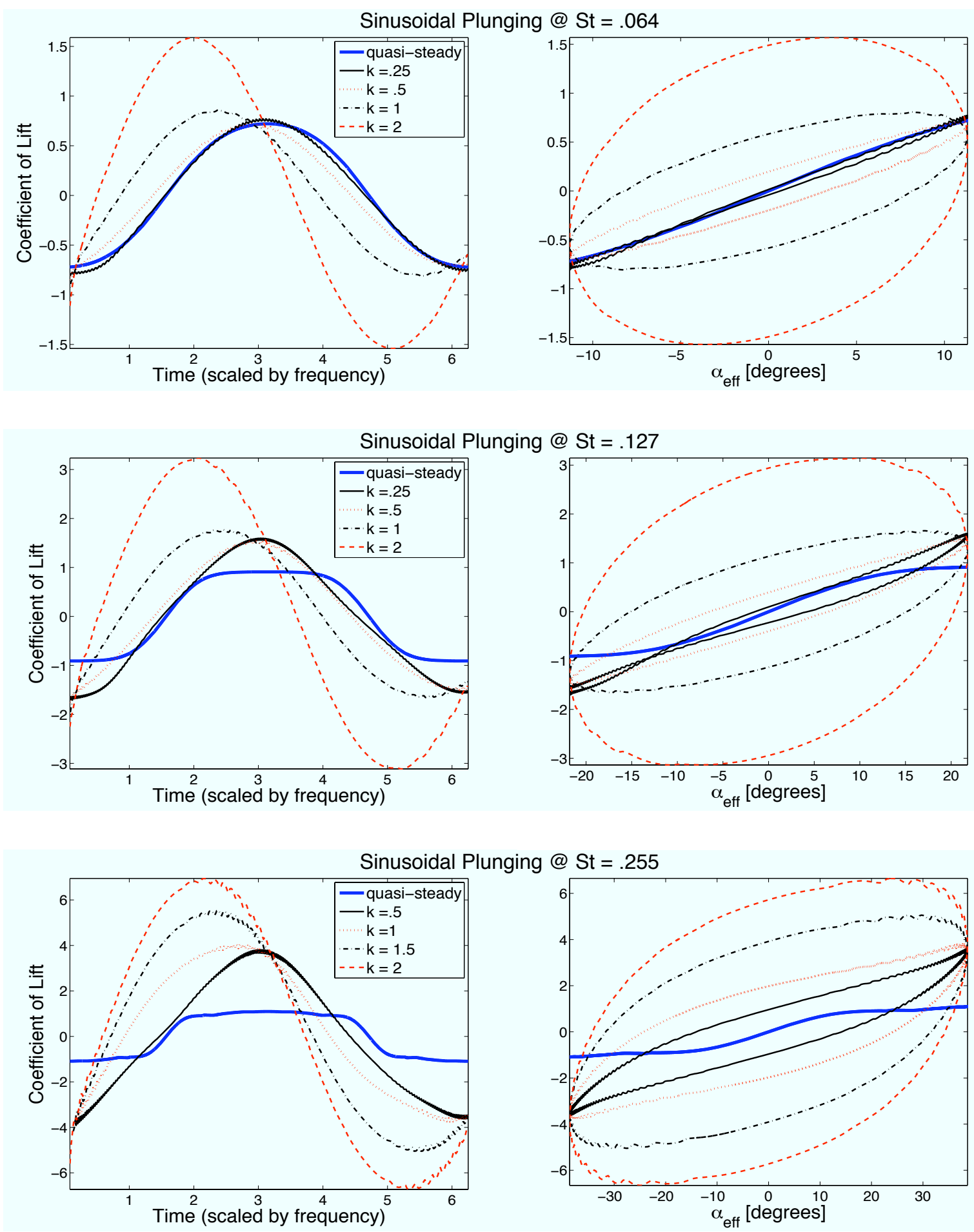

Figure 2. Comparison of actual $C_{L}$ vs. $\alpha_{\text {eff }}$ approximation for plate in sinusoidal plunge maneuver at $S t \in\{.064, .127, .255\}$. Center of mass of plate is $h(t)=(A / 2) \sin (2 \pi f t)$; Strouhal number is $\mathbf{S t}=f A / U$; reduced frequency is $k=2 \pi f c /(2 U)$. Deviation from quasi-steady occurs at $k \approx 0.5$. (Left) $C_{L}$ vs. time (Right) $C_{L}$ vs. $\alpha_{\text {eff }}$. 


\section{Lagrangian coherent structure visualizations}

To begin characterizing the fundamental unsteady fluid dynamics of airfoils at low Reynolds numbers, it is necessary to identify separated regions and wake structures. For an unsteady flow field, it is not possible to identify separated regions with streamlines alone. Instead, we use Lagrangian coherent structures ${ }^{13,14}$ (LCS), which are computed from a time-resolved sequence of snapshots of an unsteady flow. These LCS are ridges in the field of Finite Time Lyapunov Exponents (FTLE), and indicate regions of large stretching between nearby particles. FTLE fields may be computed using backward (resp. forward) time integration, in which case the LCS represents attracting (resp. repelling) material lines and isolate regions of separated flow around the plate and in the wake. In the following visualizations we exclusively use FTLE fields measuring stretching in backward time to obtain structures which are attracting in forward time. LCS for four interesting cases are shown in Figure 3. The validation of the LCS as attracting material lines is shown in the Appendix.

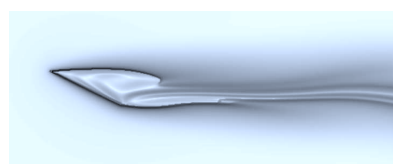

Static 25

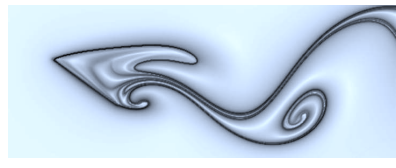

Static 35
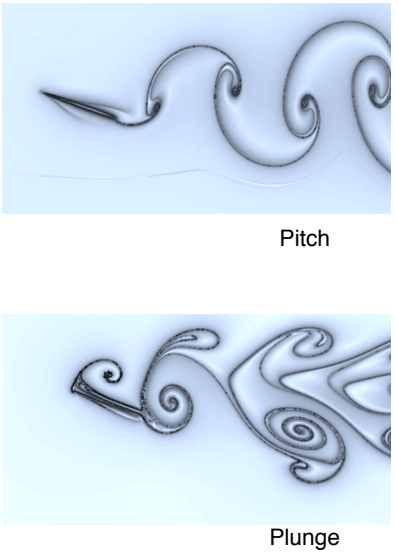

Figure 3. Lagrangian coherent structures for a variety of stationary and moving plates. (Top Left) Stationary plate at $\alpha=25^{\circ}$ (Bottom Left) Stationary plate at $\alpha=35^{\circ}$ (Top Right) Pitching plate (Bottom Right) Plunging plate with $20^{\circ}$ bias.

Figure 4 shows LCS visualizations of the formation and convection of a leading-edge vortex (LEV) on a flat plate in periodic pure-pitch and pure-plunge maneuvers. Understanding the role of the the LEV and dynamic stall vortex (DSV) on the lift, drag and moment of a rapidly maneuvering airfoil is essential to building a set of physical models suitable for control even in the presence of large disturbances and transients. Moreover, LCS illustrate structures in flow fields of interest such as the point of high lift in a post-stall airfoil which will lend insight into the design of model-based controls to steer the flow into desired states. It is interesting to note the similarity between the Lagrangian Coherent Structures shown in Figures 3 and 4 and classic flow visualizations, such as those of Koochesfahani ${ }^{15}$.

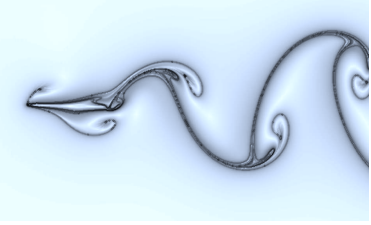

Pitch

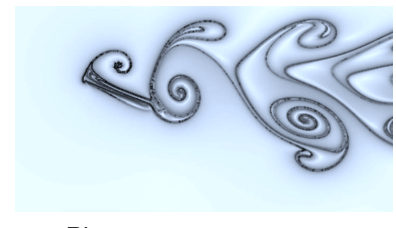

Plunge
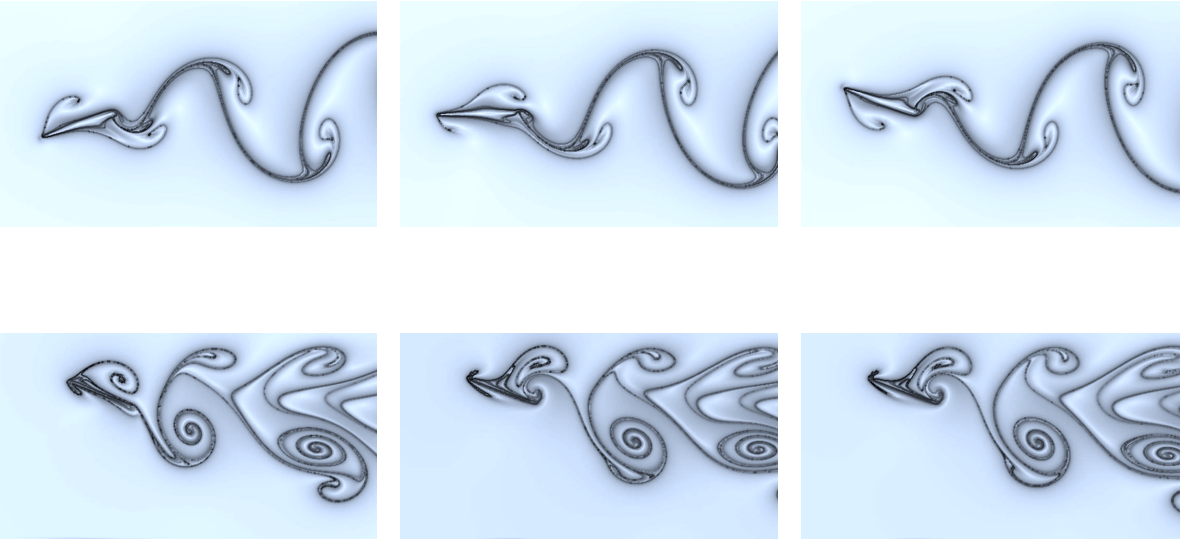

Figure 4. Visualization of leading-edge vortex (LEV) formation and convection using Lagrangian coherent structures (LCS). (Top) Plate pitching about trailing edge with amplitude of $20^{\circ}$, Strouhal number .342 and reduced frequency $k=.25$ (Bottom) Plate with $20^{\circ}$ bias plunging with Strouhal number .3 and reduced frequency $k=.25$. 


\section{Semi-empirical nonlinear models}

A simple system of differential equations which describes the transient and steady-state lift associated with an impulsively started 2D plate at a fixed angle of attack includes a Hopf bifurcation and a decoupled first-order lag

$$
\left.\begin{array}{l}
\dot{x}=\left(\alpha-\alpha_{c}\right) \mu x-\omega y-a x\left(x^{2}+y^{2}\right) \\
\dot{y}=\left(\alpha-\alpha_{c}\right) \mu y+\omega x-a y\left(x^{2}+y^{2}\right) \\
\dot{z}=-\lambda z
\end{array}\right\} \Longrightarrow \begin{aligned}
& \dot{r}=r\left[\left(\alpha-\alpha_{c}\right) \mu-a r^{2}\right] \\
& \dot{\theta}=\omega \\
& \dot{z}=-\lambda z
\end{aligned}
$$

The $z$ direction is decoupled and represents the exponential decay of transient lift generated from the impulsive start. Transforming the $(x, y)$ system into polar coordinates, it becomes clear that there is a fixed point at $r=0$. This fixed point undergoes a subcritical Hopf bifurcation at $\alpha=\alpha_{c}$ resulting in an unstable fixed point at $r=0$ and a stable limit cycle with radius $R=\sqrt{\left(\alpha-\alpha_{c}\right) \mu / a}$. The limit cycle represents the fluctuations in lift due to periodic vortex shedding of a plate at an angle of attack which is larger than the stall angle. Thus, at a particular angle of attack $\alpha$, the unsteady coefficient of lift $C_{L}$ is constructed from the average lift $\bar{C}_{L}$ and the state variables $y$ and $z$ as follows

$$
C_{L}=\bar{C}_{L}+y+z
$$

With knowledge of the actual lift vs. time from numerical experiment, it is possible to tune the parameters $(\mu, \lambda, \omega, a)$ with the rates of decay, period of shedding and amplitude of stable lift fluctuations. Initial conditions of the model are chosen to start the system with the right transient lift and phase. By properly tuning the constants with experimental data, this model will closely reproduce the transient lift dynamics of a stationary plate for a wide range of angles of attack $\alpha$. Figure 5 shows a typical example, for $\alpha=35^{\circ}$.

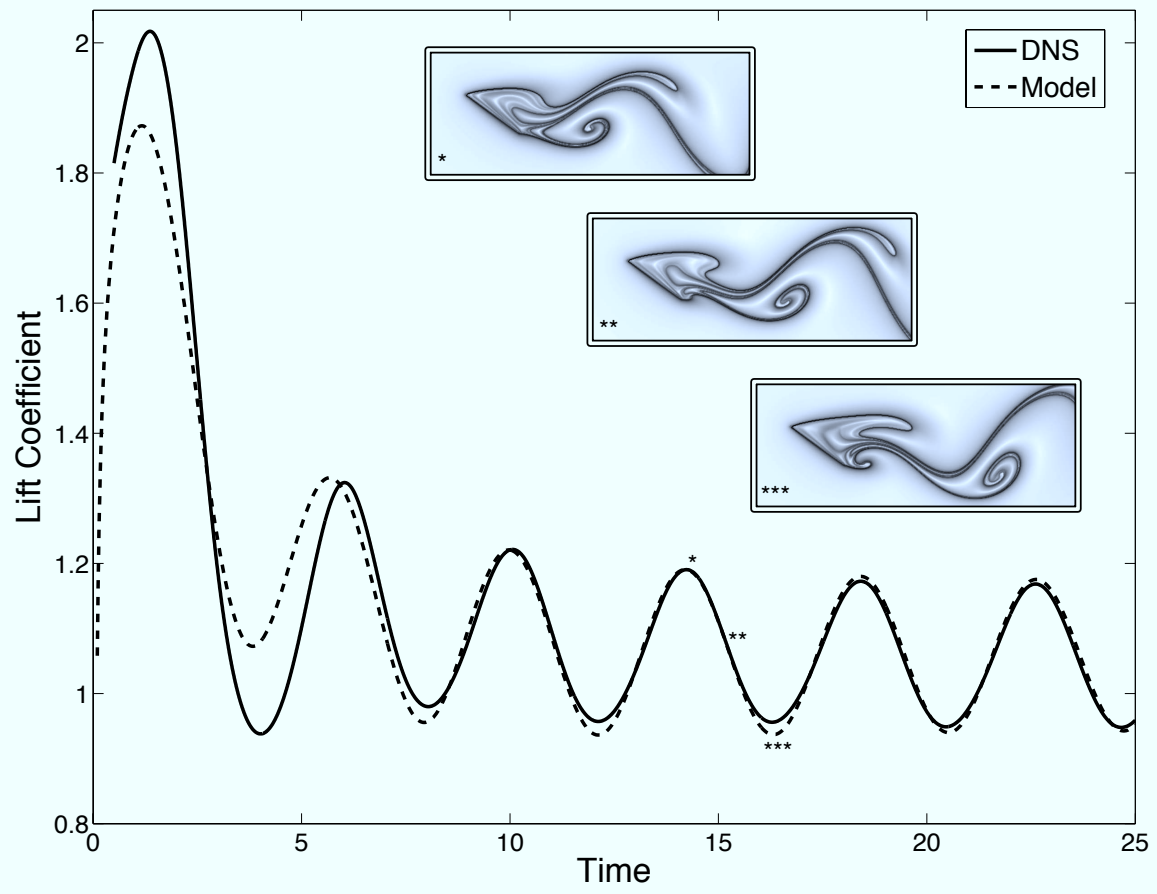

Figure 5. Coefficient of lift vs. time for stationary plate at post-stall angle $\alpha=35^{\circ}$. The solid line represents the oscillating lift curve obtained from direct numerical simulation and the dashed line is a the output of a low order ODE model with a Hopf bifurcation and a lag. The inset panels show coherent structures in the flow field at instances of high, moderate and low lift.

It is important to note that the model (1) is specifically chosen to exhibit a supercritical Hopf bifurcation as the angle of attack $\alpha$ varies through $\alpha_{c}$. Such a bifurcation is observed in simulations and experiments (Figure 6), so for a fixed set of parameters, the model (1) may be tuned to match the observed behavior over a range of angle of attack $\alpha$. Theoretically, one would expect such a model to be valid only for values of $\alpha$ close to the bifurcation value $\alpha_{c}$, but in practice we have observed that, even for fixed parameters, the model (1) may be tuned to match simulations reasonably closely over a wide range of parameter values, from $\alpha=0$ to at least $35^{\circ}$. 


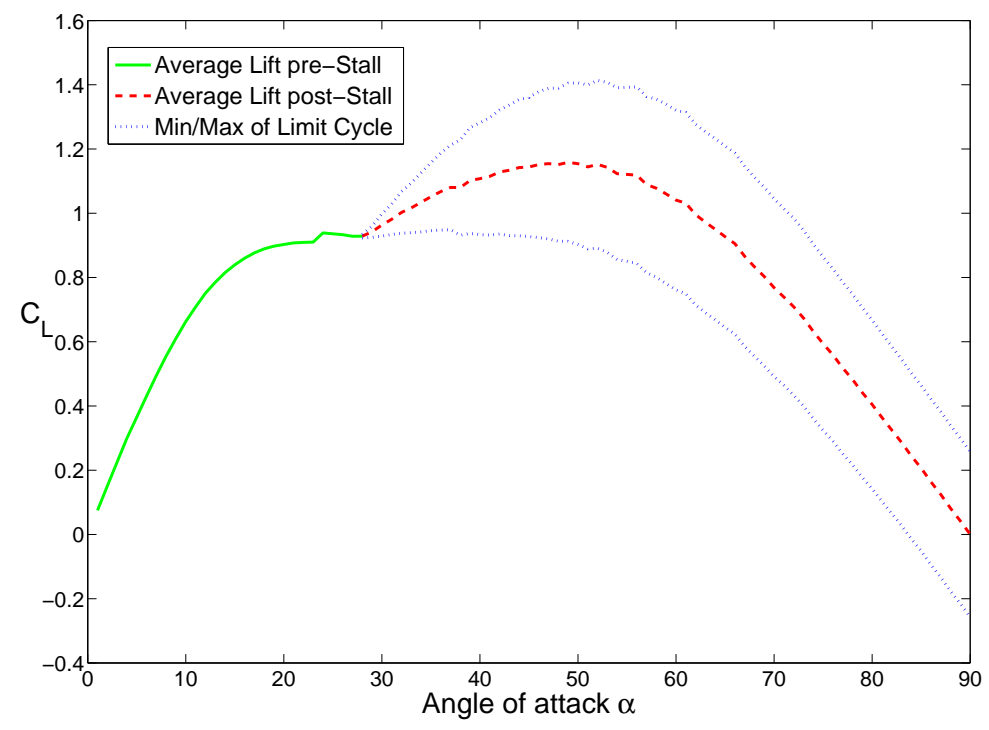

Figure 6. Lift vs. angle of attack for fixed plate at $R e=100$. Hopf bifurcation occurs at $\alpha \approx 28^{\circ}$. Dashed line represents average post-stall lift and dotted lines represent minimum and maximum post-stall lift.

It also is possible to model the transient lift from the impulsive start by coupling $z$ and $(x, y)$ :

$$
\left.\begin{array}{rl}
\dot{x} & =\left(\alpha-\alpha_{c}\right) \mu x-\omega y-a x z \\
\dot{y} & =\left(\alpha-\alpha_{c}\right) \mu y+\omega x-a y z \\
\epsilon \dot{z} & =-z+\left(x^{2}+y^{2}\right)
\end{array}\right\} \Longrightarrow \begin{aligned}
\dot{r} & =r\left[\left(\alpha-\alpha_{c}\right) \mu-a z\right] \\
\dot{\theta} & =\omega \\
\epsilon \dot{z} & =-z+r^{2}
\end{aligned}
$$

For $\epsilon \ll 1$, trajectories quickly settle to the "slow" manifold $z=x^{2}+y^{2}$, which reduces to our original dynamics. This method has been useful in characterizing transients in the wake of a cylinder ${ }^{16}$. Because the flat plate at high angles of attack is a bluff body, its wake topology should be structurally equivalent to the wake behind a cylinder.

The models (1) and (2) capture reasonable dynamic behavior, but they have a severe limitation for the present application, since the initial values of the states $(x, y, z)$ need to be carefully chosen in order to match the transient lift response. In practice, this transient response is precisely what we are interested in modeling, but these initial conditions are not known. Instead, these states are excited by external factors, such as disturbances or changes in angle of attack $\alpha$ (which can lead to the formation of leading-edge vortices, as stated earlier). One idea for incorporating these effects is to add impulsive forcing to the model $\dot{\mathbf{x}}=\ldots+\mathbf{f}(\alpha, \dot{\alpha})$, so that the terms $\mathbf{f}$ can excite the states $\mathbf{x}$ through a change of angle of attack. Such models are the subject of our present work.

\section{Three-dimensional simulations}

The three-dimensional results discussed here are preliminary in nature and are intended to highlight the similarities and differences between the two- and three-dimensional flows. Emphasis is placed upon introducing the overall vortex dynamics behind a rectangular plate undergoing a pitch-up motion.

We simulate flow behind a rectangular plate of $A R=2$, in a pitch-up motion using the three-dimensional immersed-boundary projection method of Taira and Colonius ${ }^{10}$. First, to obtain a suitable initial condition for simulating the pitching plate, the plate is instantaneously materialized (or impulsively translated) in uniform flow and is kept at zero angle of attack. The Reynolds based on the freestream velocity and chord length is set to $R e=U_{\infty} c / \nu=300$. The simulation is performed for 40 convective time units to allow the flow field to reach a steady state, which is then selected as the initial condition for the pitching plate study. With this initial condition, we now gently pitch the plate from $\alpha=0^{\circ}$ to $30^{\circ}$ about the mid-chord in the following fashion:

$$
\alpha(t)=\alpha_{\max } \frac{1}{2}\left[1+\operatorname{erf}\left(\frac{t-t_{a}}{\sqrt{2} \sigma}\right)\right] \text { and } \quad \dot{\alpha}(t)=\alpha_{\max } \frac{1}{\sqrt{2 \pi} \sigma} \exp \left[-\frac{\left(t-t_{a}\right)^{2}}{2 \sigma^{2}}\right],
$$

where $\alpha_{\max }=30^{\circ}, t_{a}=3 \sigma$ and $\sigma=3$ are chosen. The motion is considered to be completed after $t=2 \times 3 \sigma=18$ from the initial condition. In order to focus on the formation of tip vortices and the interaction of wake vortices, we only consider the plate to go under a pitch up motion, which can be thought of a model for a half-cycle of an entire pitching period. Further details on the numerical setup and validation are provided in Taira and Colonius ${ }^{10}$. 


\section{A. 3D flow profile}

Snapshots in Figure 7 illustrate the evolution of the vortical wake during the pitch-up process. As the plate starts to pitch up, a low pressure region is created above the top surface keeping the vortical structure from the leading edge closely attached. Concurrently, the tip vortices are formed by rolling the vortex sheets from the tip and trailing edges. Transient features of the tip vortices show up with spiraling motion which eventually decay with the loss of the columnar structure of the tip vortices. As the tip vortices decrease their strengths, the downward induced velocity pressing down the leading-edge vortex weakens its influence and allows the leading-edge vortex to separate and shed into the wake.

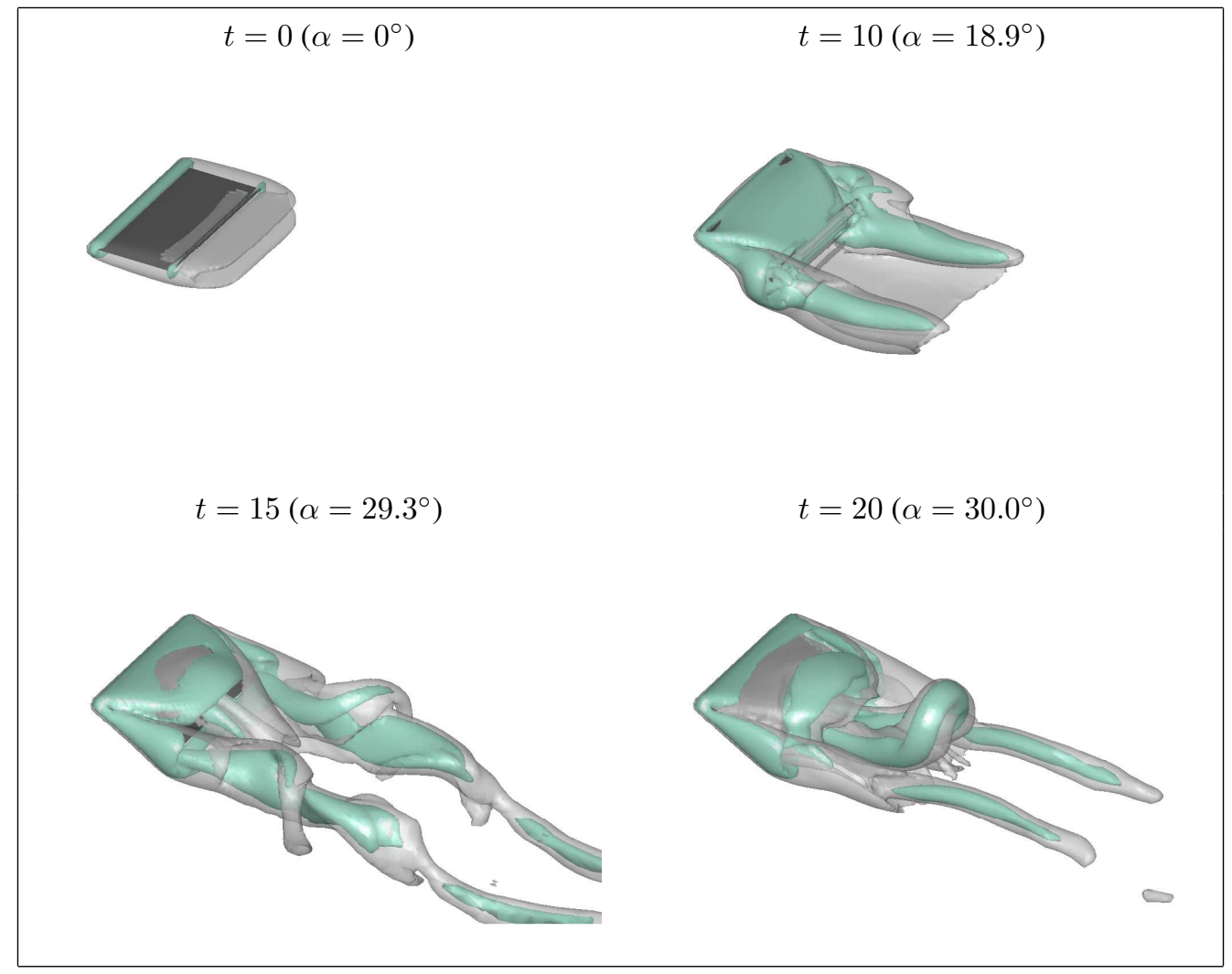

Figure 7. Snapshots of the wake evolution behind a rectangular flat plate of $A R=2$ pitching from $\alpha=0^{\circ}$ to $30^{\circ}$ at $R e=300$. Isosurfaces of $\|\boldsymbol{\omega}\|=3$ and $Q=3$ are shown in gray and aquamarine, respectively. Viewing downward from the port side of the plate.

The corresponding lift and drag coefficients of the plate are shown in Figure 8. It can be seen that the maximum lift is achieved when a large leading-edge vortex is closely formed above the plate at $t \approx 10$. This time also coincides with the time at which the maximum angular velocity is experienced by the plate for this example. As the vorticity accumulates, a hairpin vortex is formed between $t=12-15$ which eventually is released into the wake around $t \approx 20$. During this shedding stage, the lift is significantly decreased due to the loss of the spanwise circulation and the lowpressure region above the plate.

Compared to the two-dimensional flows, the three-dimensional analogs have significant influence from the tip vortices in stabilizing the attachment of the leading-edge vortex. In a complete pitching motion, tip vortices will interact strongly with the wake vortices while affecting the attachment of the leading-edge vortex ${ }^{* *}$. Despite the differences, the force exerted on the low-aspect-ratio plate shows a trend similar to the two-dimensional flow. The sharp increase in lift and drag are observed during the pitch-up phase from the existence of the leading-edge vortex followed by a decrease in lift due to the loss thereof. In the three-dimensional case, there is a secondary peak generated (around $t \approx 12.5$ ) in the force coefficients following the maximum, due to the interaction of the leading-edge and tip vortices. Since this fluctuation is much smaller than the dominant peak, its secondary effect may not be much of a concern. Hence, it is expected that the previous two-dimensional low-order model can be extended to capture the qualitative force history. In order to capture secondary effects resulting from the interaction of wake vortices, it may be necessary to increase the number of terms but again would be of minor correction to the overall model.

\footnotetext{
${ }^{* *}$ In pure translating flights, strengthening the tip vortices with actuation were found to enhance lift. Strong downward induced velocity from the tip vortices retained the LEV closer to the top surface over time ${ }^{10}$.
} 


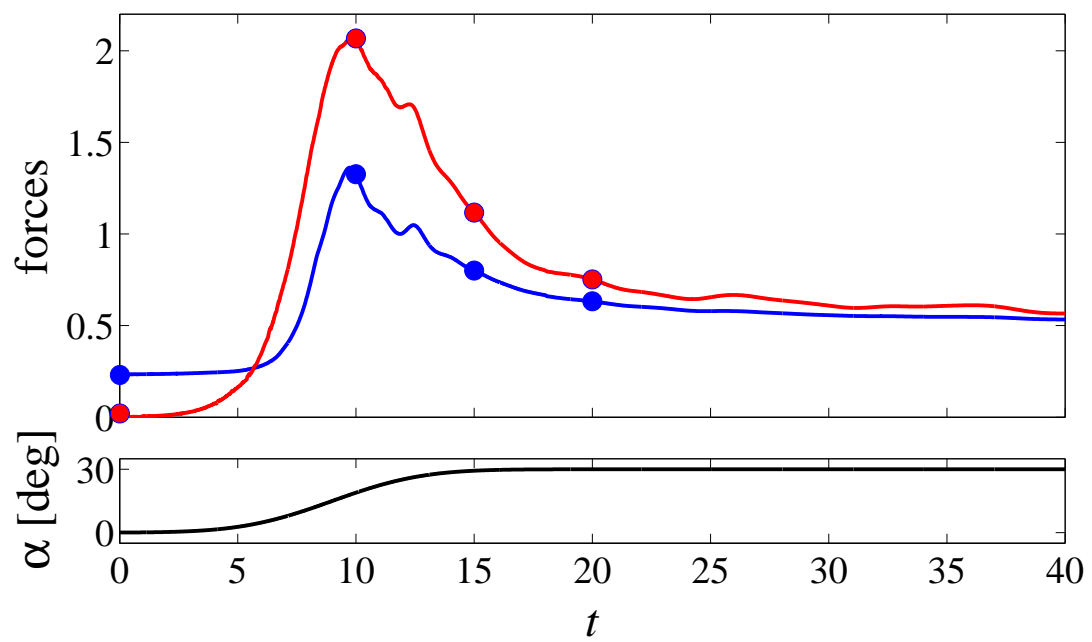

Figure 8. Lift and drag coefficients for the rectangular plate of $A R=2$ over time, shown by the red and blue lines, respectively. Overlaid circles denote the time at which snapshots are available in Figure 7. Bottom plot shows the corresponding time trace of the angle of attack of the plate.

\section{Wind tunnel experiments}

A state-space model capable of representing certain aircraft characteristics at high angles of attack was presented by Goman and Khrabrov in $1995^{8}$. The model was useful in representing the dynamic effects on lift associated with the dynamic stall vortex produced during rapid pitching maneuvers. We are interested in exploring how well this model fits the measurements of lift obtained from a semi-circular wing model pitching in a wind tunnel, and if some physical insight can be gained.

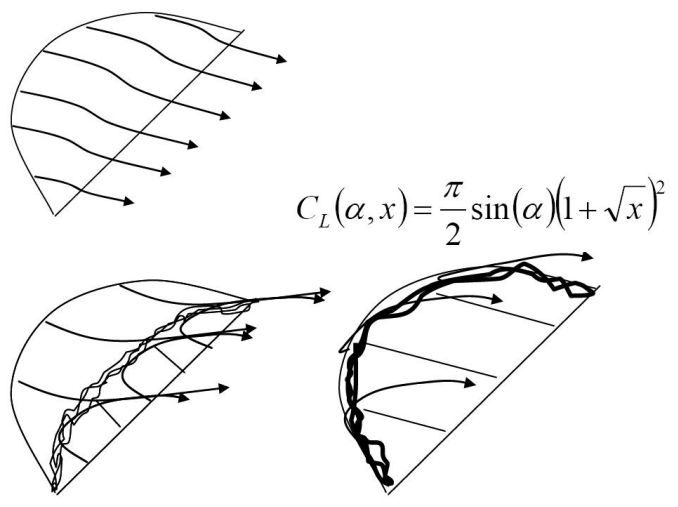

Figure 9. Partial separation develops with increasing angle of attack. The variable $x$ represents the degree of flow attachment.

The underlying concept of the G-K model is to represent the degree of flow attachment by a parameter $x$, in which $x=1$ represents fully attached flow, and $x=0$ represents fully separated flow. The sketches in Figure 9 illustrate how the fully attached flow at low angles of attack $(x=1)$ evolve into a fully separated flow $(x=0)$ as the angle of attack of the wing increases. The lift coefficient is written as a function of angle of attack $\alpha$, and the attachment parameter $x$. The state space model for the lift coefficient is written as

$$
\begin{gathered}
\tau_{1} \frac{d x}{d t}+x=x_{0}\left(\alpha-\tau_{2} \dot{\alpha}\right) \\
C_{L}(\alpha, x)=0.61 \frac{\pi}{2} \sin \alpha(1+\sqrt{x})
\end{gathered}
$$

where $\tau_{1}$ and $\tau_{2}$ are time constants used to fit the data. The constant $\tau_{1}$ can be interpreted as the relaxation time for the lift coefficient to respond to a change in angle of attack. The constant $\tau_{2}$ is a time delay related to the dynamic motion of the wing. The function $x_{0}(\alpha)$ is the degree of separation measured in static tests as the angle of attack is varied over 
a wide range. Figure 10 shows the measured static function $x_{0}(\alpha)$ as the blue line on the left side of the figure. The effect of the time delay $\tau_{2}=0.1$ on $x$ is shown by the red line in the middle. The effect of the setting the relaxation time $\tau_{1}=0.1$ is illustrated by the green line on the far right side of the figure. The constant 0.61 is a correction to the lift-curve slope related to finite span wing effects.

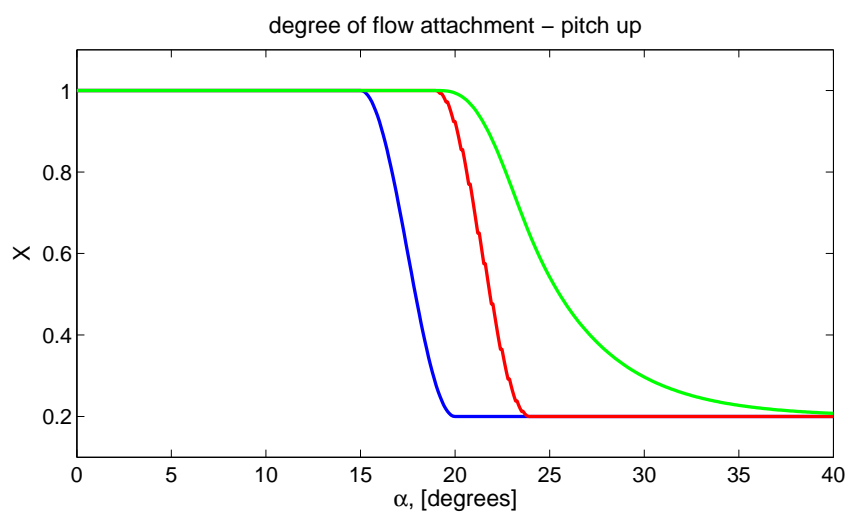

Figure 10. Static flow attachment function $x_{0}(\alpha)$ (blue, leftmost curve); shift to higher angles associated with $\tau_{2}$ delay (red, middle curve); relaxation and shift associated with $\tau_{1}$ and $\tau_{2}$ (green, rightmost curve).

\section{A. Single pitch-up maneuver}

The wing was pitched up in a single sweeping motion from $\alpha=-40^{\circ}$ to $+40^{\circ}$ at three different pitch rates. One was a very slow pitch rate of $0.5 \mathrm{deg} / \mathrm{s}$ simulating quasi-static behavior. The second and third pitch rates were at $40 \mathrm{deg} / \mathrm{s}$ and $80 \mathrm{deg} / \mathrm{s}$, respectively. The experimental results and comparison with the G-K model are shown in Figure 11. The blue, green and red lines correspond to the $0.5,40$ and $80 \mathrm{deg} / \mathrm{s}$ pitch rates. A comparison with the G-K model is also shown using $\tau_{1}=0.02 \mathrm{~s}$ and $\tau_{2}=0.06 \mathrm{~s}$. The experimentally measured lift curves are shifted slightly to the right relative to the G-K model predictions. The shift is consistent with a change in the relative angle of attack associated with the rotation of the leading edge of the wing. Reasonably good agreement between the maximum lift coefficient and the relaxation after complete flow separation in the dynamic cases was found.

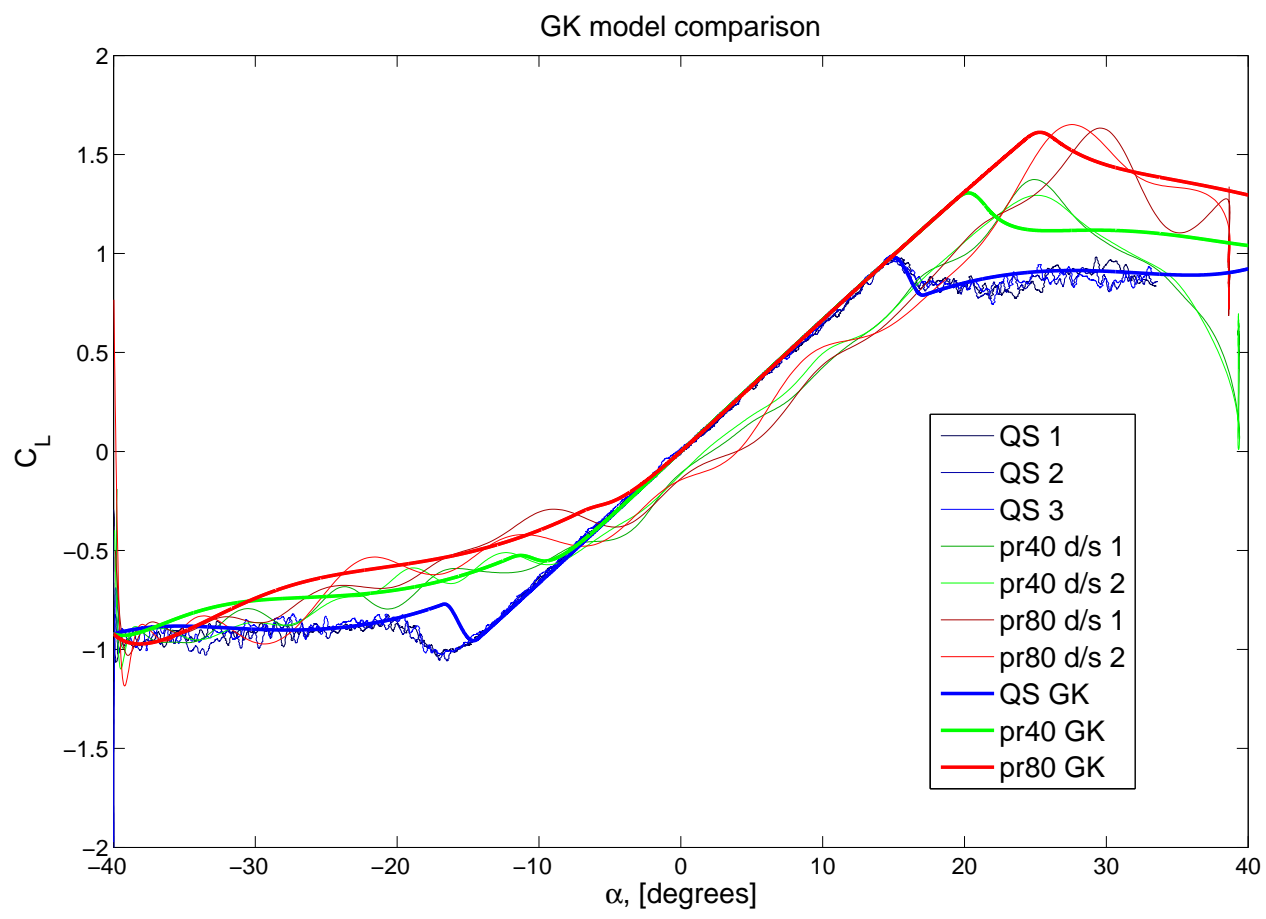

Figure 11. Single pitch-up maneuver from $\alpha=-40^{\circ}$ to $+40^{\circ}$, showing quasisteady results (QS), and pitch rates of 40 and $80 \mathrm{deg} / \mathrm{sec}$, comparing experimental data with results from the calibrated Goman-Khrabrov model (indicated by GK). 


\section{B. Oscillating pitch maneuver}

In the oscillating pitch maneuver the wing is positioned at a mean angle of $\alpha=19^{\circ}$, then oscillated with a \pm 4 degree amplitude. The experiment and G-K model are compared in Figure 12 for an wing oscillation frequency of $0.5 \mathrm{~Hz}$. The baseline static lift curve is also plotted for reference. Because the flow over the wing is partially separated, a hysteresis loop in the lift curve is formed. The same time constants were used in the G-K model as in the steady pitch up maneuver discussed before, except that the frequency of oscillation was increased by a factor of 4 . This is equivalent to increasing the delay time constant $\tau_{2}$ by a factor of 4 .

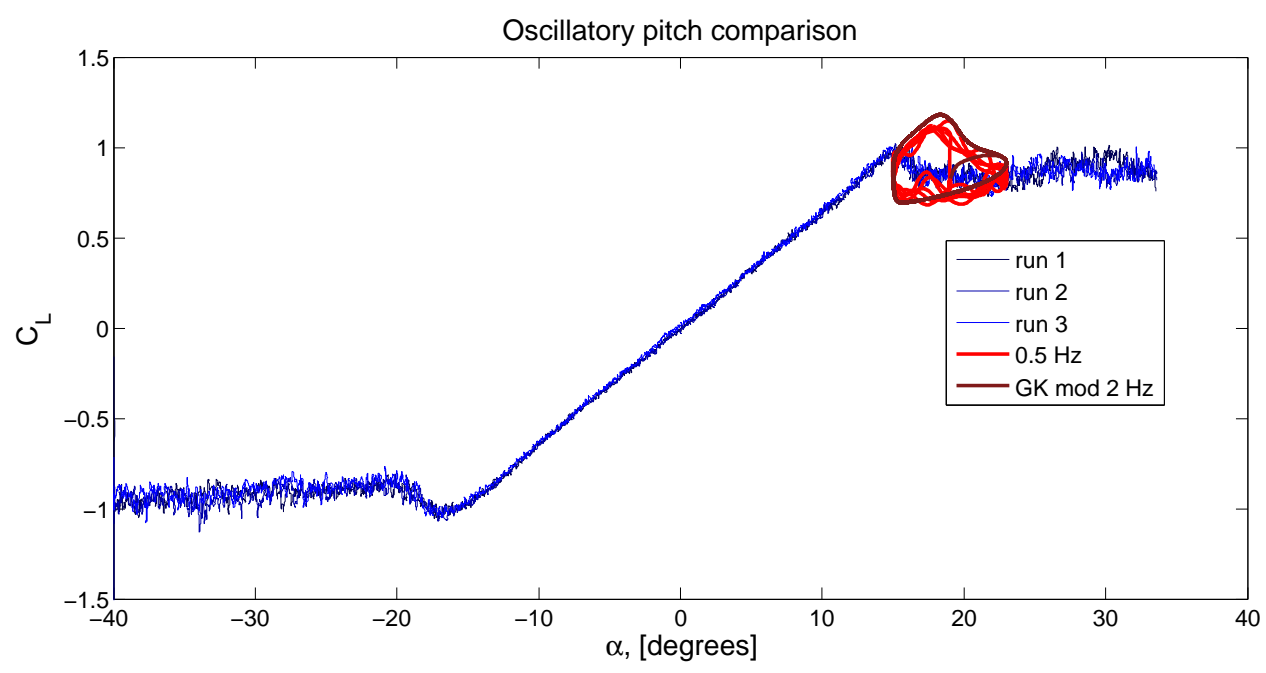

Figure 12. Oscillating at $0.5 \mathrm{~Hz}$. Red line: experiment; Brown line: G-K model assuming an "artificial" oscillation rate of $2.0 \mathrm{~Hz}$.

Figures 13 and 14 show the same type of oscillatory wing motion, but at higher frequencies of $1 \mathrm{~Hz}$ and $2 \mathrm{~Hz}$, respectively. A significant change in the shape of the hysteresis loop occurs as the frequency increases. The changes in shape of the hysteresis loops could be mimicked by the G-K model, but it was necessary to increase the delay time constants by a factor of 4 and 6 for the $1.0 \mathrm{~Hz}$ and $2.0 \mathrm{~Hz}$ oscillation cases.

The ability of the model to capture the general features of the lift hysteresis suggests that time delays and relaxation times play a major role in determining the instantaneous forces acting on the maneuvering wing. The fact that the delay time constant needed to be changed to fit the specific case indicates the model is incomplete, and suggests the physics responsible for the time delay are different between the single pitch maneuver and the oscillating wing maneuver.

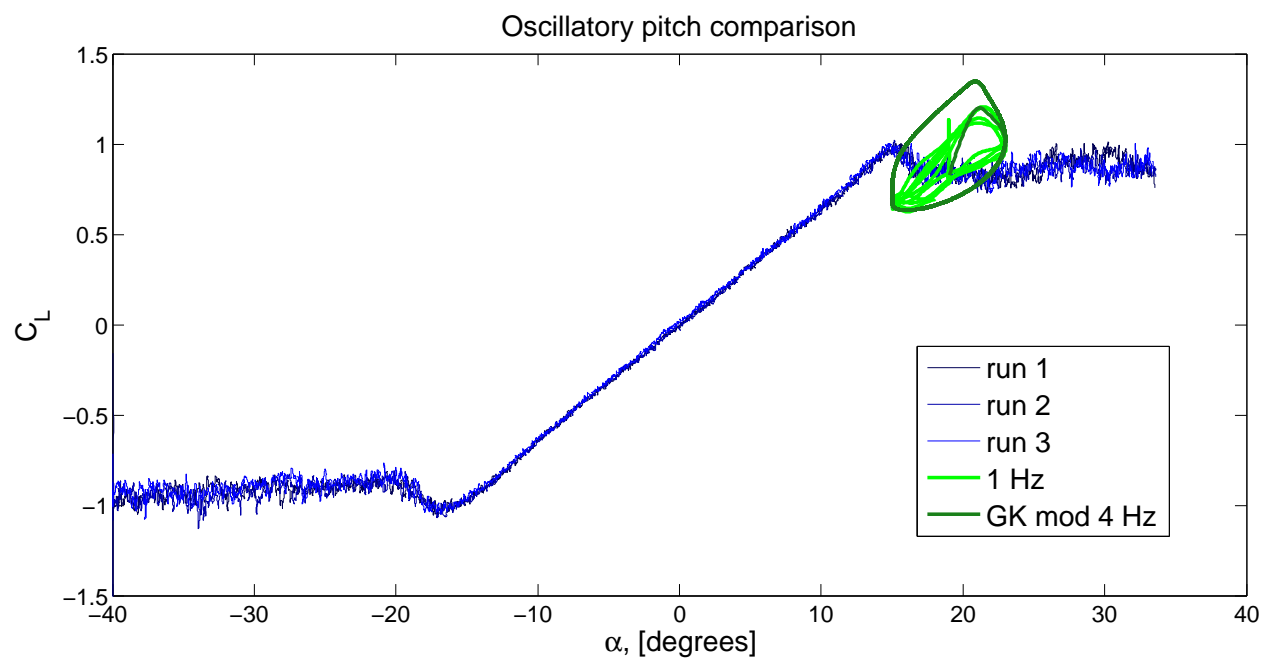

Figure 13. Oscillating at 1.0 Hz. Light green: experiment; Dark green: G-K model assuming an "artificial” oscillation rate of 4.0 Hz. 


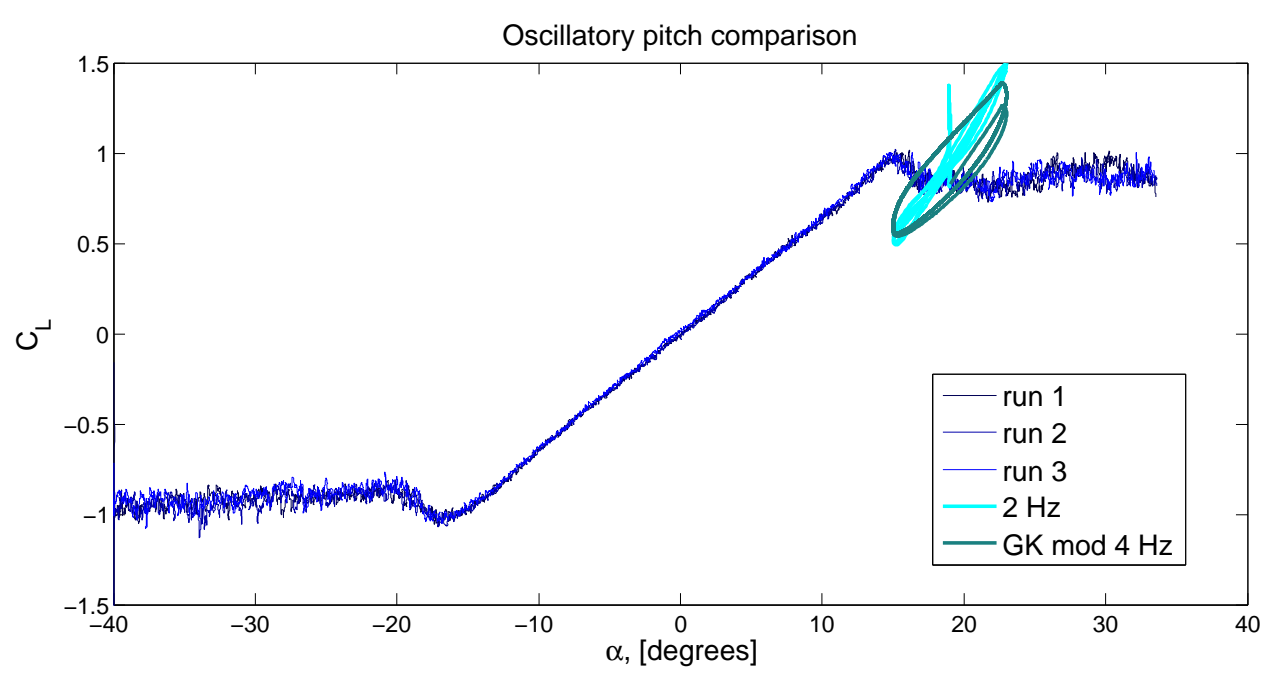

Figure 14. Oscillating at $2.0 \mathrm{~Hz}$. Light blue: experiment; Dark blue: G-K model assuming an "artificial" oscillation rate of $12.0 \mathrm{~Hz}$.

\section{Conclusions}

We have investigated the unsteady forces on low-Reynolds number pitching wings using 2D and 3D direct numerical simulations, and wind tunnel experiments. The onset of unsteadiness in a sinusoidally plunging plate with $A R=\infty$ has been identified with a reduced frequency $k=0.5$ over a range of moderate Strouhal numbers. At first glance, the independence on Strouhal number may be surprising, but one would expect that the reduced frequency $k$ should be the most important parameter for determining when it is critical to model the unsteadiness, since it relates the timescale of the motion to the particle advection time. Note that when $k<0.5$, the period of the motion is longer than the time it takes a particle to convect one chord length, so that the flow has time to relax into quasi-steady state. Additionally, we have visualized the formation and convection of a leading-edge vortex in sinusoidal pitch and plunge maneuvers. We have also determined the stall angle of a stationary flat plate and have used the computed average, maximum and minimum coefficient of lift and frequency of vortex shedding for $\alpha \in[0,90]$ to construct a three-state nonlinear ODE model which agrees well with the transient and steady state lift on an impulsively started plate at fixed angle. Although this model must be tuned using the parameters extracted from experiments, it shows that because the behavior is deterministic, it is possible to construct a relatively low order model which agrees very well with data.

DNS for a 3D pitch-up maneuver of a rectangular plate with $A R=2$ shows similarities and differences between $2 \mathrm{D}$ and $3 \mathrm{D}$ results. For instance, the stabilizing influence of tip vortices on the attachment of the leading-edge vortex is unique to 3 dimensional wings. However, the force exerted on a low-aspect-ratio plate in a pitch-up maneuver is qualitatively similar to the force on an impulsively started 2D plate, suggesting that the low-order model constructed for 2D impulsively started wings may be extended.

Finally, wind tunnel data for a semi-circular wing in single pitch-up and sinusoidal pitch maneuvers was compared with an empirical model proposed by Goman and Khrabrov ${ }^{8}$ to demonstrate the importance of time delays and relaxation times in determining the forces on a maneuvering wing. An interesting feature of the G-K model is that it is based on a physical variable, the degree of flow attachment $x$, and physical time scales. Models based on relevant physical variables, such as attachment point or vortex size, strength, and aspect ratio will be important in determining the relationship between excitation/relaxation time scales and a particular maneuver at a given state.

It will be interesting to extend this analysis in a number of directions. As in the case of a sinusoidally plunging plate, it is possible to relate the lift of a sinusoidally pitching plate to that of a stationary plate with an effective camber. It is also possible to extend the low-order model to capture the coefficients of moment and $\operatorname{drag} C_{D}, C_{M}$ for static attack angles and compare the model for maneuvers which are known to have hysteresis, as in the G-K model comparison. In addition to analyzing backward time LCS (forward time attracting sets) it will be useful to visualize forward time LCS (forward time repelling sets) and to extend the LCS computation to 3D flow fields. This will provide a more complete picture of flow structures and lead to a better understanding of the physical mechanism underlying these transient flow events. It will also be interesting to extend our low order model to include physically relevant variables such as leading and trailing-edge vortex strength and position. 


\section{Appendix}
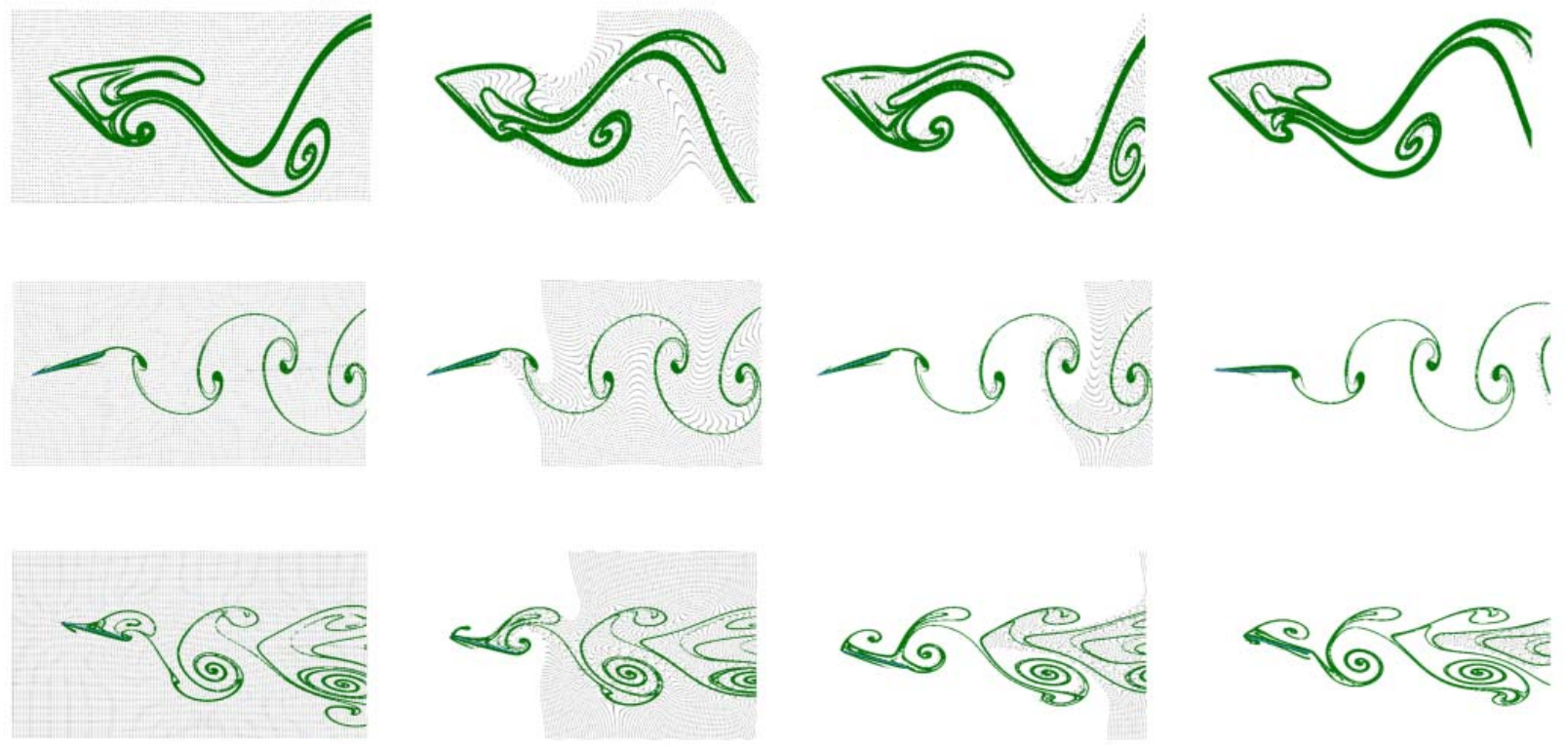

Figure 15. Validation of Lagrangian coherent structures with a grid of points integrated forward along velocity field snapshots. Time and flow move from left to right. Slow particles collect on these attracting lines. (Top) Stationary plate at $\alpha=35^{\circ}>\alpha_{\text {crit }}$ (Middle) Plate pitching about quarter-chord with amplitude of $20^{\circ}$, Strouhal number .2565 and reduced frequency $k=.25$ (Bottom) Plate with $20^{\circ}$ bias plunging with Strouhal number .3 and reduced frequency $k=.25$.

\section{References}

${ }^{1}$ Smith, A., Vortex models for the control of stall., PhD thesis, Boston University, 2005.

${ }^{2}$ Magill, J., Bachmann, M., Rixon, G., and McManus, K., "Dynamic stall control using a model-based observer." J. Aircraft, Vol. 40, No. 2, 2003, pp. 355-362.

${ }^{3}$ Birch, J. and Dickinson, M., "Spanwise flow and the attachment of the leading-edge vortex on insect wings." Nature, Vol. 412, 2001, pp. 729-733

${ }^{4}$ Sane, S., "The aerodynamics of insect flight.” J. Exp. Biol., Vol. 206, No. 23, 2003, pp. 4191-4208.

${ }^{5}$ Videler, J., Samhuis, E., and Povel, G., "Leading-edge vortex lifts swifts.” Science, Vol. 306, 2004, pp. 1960-1962.

${ }^{6}$ Ahuja, S., Rowley, C., Kevrekidis, I., Wei, M., Colonius, T., and Tadmor, G., "Low-dimensional models for control of leading-edge vortices: Equilibria and linearized models." AIAA Aerospace Sciences Meeting and Exhibit, 2007.

${ }^{7}$ Rowley, C., "Model reduction for fluids using balanced proper orthogonal decomposition." Int. J. Bifurcation Chaos, Vol. 15, No. 3, 2005, pp. 997-1013.

${ }^{8}$ Goman, M. and Khabrov, A., "State-space representation of aerodynamic characteristics of an aircraft at high angles of attack." J. Aircraft, Vol. 31, No. 5, 1994, pp. 1109-1115.

${ }^{9}$ Taira, K. and Colonius, T., "The immersed boundary method: a projection approach.” J. Comput. Phys., Vol. 225, No. 2, 2007, pp. 21182137.

${ }^{10}$ Taira, K., Dickson, W., Colonius, T., Dickinson, M., and Rowley, C., "Unsteadiness in flow owver a flat plate at angle-of-attack at low Reynolds numbers." AIAA Aerospace Sciences Meeting and Exhibit, 2007.

${ }^{11}$ Sears, W., "Some aspects of non-stationary airfoil theory and its practical applications." AIAA Journal Special Supplement: Centennial of Powered Flight, Vol. 8, No. 3, 1941, pp. 104-108.

${ }^{12}$ von Karman, T. and Sears, W., "Airfoil theory for non-uniform motion.” J. Aeronautical Sciences, Vol. 5, No. 10, 1938, pp. 379-390.

${ }^{13}$ Green, M., Rowley, C., and Haller, G., "Detection of Lagrangian coherent structures in 3D turbulence." J. Fluid Mech., Vol. 572, 2007, pp. $111-120$

${ }^{14}$ Shadden, S., Lekien, F., and Marsden, J., "Definition and properties of Lagrangian coherent structures from finite-time Lyapunov exponents in two-dimensional aperiodic flows." Physica D, Vol. 212, No. 34, 2005, pp. 271-304.

${ }^{15}$ Koochesfahani, M. M., "Vortical patterns in the wake of an oscillating airfoil," AIAA J., Vol. 27, 1989, pp. 1200-1205.

${ }^{16}$ Noack, J., Afanasiev, K., Morzynski, M., Tadmor, G., and Thiele, F., "A hierarchy of low-dimensional models for the transient and posttransient cylinder wake.” J. Fluid Mech., Vol. 497, 2003, pp. 335-363. 